\title{
Structural trends in clusters of quadrupolar spheres
}

\author{
Mark A. Miller, James J. Shepherd, and David J. Wales \\ University Chemical Laboratories, Lensfield Road, Cambridge CB2 1EW, United Kingdom
}

(Dated: November 15, 2018)

\begin{abstract}
The influence of quadrupolar interactions on the structure of small clusters is investigated by adding a point quadrupole of variable strength to the Lennard-Jones potential. Competition arises between sheet-like arrangements of the particles, favoured by the quadrupoles, and compact structures, favoured by the isotropic Lennard-Jones attraction. Putative global potential energy minima are obtained for clusters of up to 25 particles using the basin-hopping algorithm. A number of structural motifs and growth sequences emerge, including star-like structures, tubes, shells and sheets. The results are discussed in the context of colloidal self-assembly.
\end{abstract}

\section{INTRODUCTION}

The structure adopted by a collection of particles is ultimately governed by the energetic interactions between the particles. It is therefore natural to ask what sort of structures are favoured by a given set of interactions, and also whether interactions can be chosen or manipulated in order to produce a particular target structure. The scope of both these questions is growing increasingly broad as it becomes possible to exert ever greater control over the shape and form of the interactions between molecular and colloidal building blocks [1]. The motivation for seeking deeper understanding in these areas is the desire to design novel materials and supramolecular structures with unusual and useful properties.

Considerable structural variety is possible even for spherical particles and isotropic interparticle potentials. Simple van der Waals interactions between inert gas atoms promote near-spherical, highly-coordinated structures, favouring icosahedral packing for clusters [2, 3] and close-packed crystals in the bulk. However, these structures can be suppressed by introducing a repulsive barrier into the pair potential at a distance close to $\sqrt{2}$ times the nearest neighbour separation [4]. In clusters, potentials of this form further promote icosahedral local order, but lead to polytetrahedral structures [5], or to less compact shapes that are either elongated or contain holes [6]. Local maxima in the pair potential can arise, for example, from the combination of short-range depletion attraction between colloidal particles with partially screened long-range Coulomb repulsion [7], in which case the accumulated charge of a growing cluster can effectively limit the size of aggregate that forms. It is also possible to favour less highly-coordinated order by careful design of isotropic pair potentials. For example, inverse statistical mechanical techniques can be used to derive isotropic potentials that favour crystalline but non-closepacked bulk structures, such as the diamond and wurtzite lattices [8].

A vast range of structures become possible when either non-spherical particles or anisotropic interactions are considered. Evolution has selected molecules with interactions that lead to the self-assembled structures that we observe in living matter, including pseudo one-dimensional filaments, two-dimensional membranes, tube-like channels and pores, and shell-like capsules. Fascinating examples in the latter category are the capsids of viruses, many of which are spheroidal and are constructed from a specific number of copies of a small number of different proteins [9]. It has long been known that the capsomers of some viruses can assemble in vitro into empty shells even in the absence of the viral genetic material [10], providing a powerful demonstration of how molecular shape and interactions dictate the structure of aggregates. For proteins, the symmetry and binding in oligomers is determined by the contacts between neighbours in the complex, and it is now becoming possible to engineer the quaternary structure by modifying the contact surface through mutations of the amino acid sequence [1].

Drawing inspiration from Nature, and encouraged by rapid advances in the synthesis of tailor-made building blocks, computational scientists are trying to understand the principles of self-assembly and how these can be used to build designed structures. Explicit models of polyhedral shell assembly have shown how difficult this objective can be 12]. While entropic and kinetic considerations are undoubtedly crucial for successful self-assembly, there is also a clear requirement for target structures to be energetically stable and kinetically accessible [13, 14]. A logical starting point is therefore to design building blocks with attractive sites or "patches" in geometries compatible with the target structure. Monodisperse discrete objects, as well as continuous structures, such as sheets, can be constructed in this way [15, 16].

Highly directional interactions can also be achieved through a non-uniform charge distribution in particles that remain neutral overall. For example, dipolar particles will endeavour to form chains, since the head-totail arrangement of two dipoles is low in energy and mechanically stable. The tendency to form chains is partially frustrated in the presence of competition for more compact arrangements arising from an isotropic van der Waals or depletion attraction [17, 18]. A simple model incorporating these features is the Stockmayer potential, consisting of particles with a Lennard-Jones (LJ) site plus a central point dipole. By adjusting the relative strength of the LJ and dipolar contributions, the energetically most stable morphology of the 13-particle Stockmayer 
cluster changes in four stages from a distorted icosahedron to a closed ring 17, 19]. For slightly larger sizes, knots, links and coils emerge [20].

Van Workum and Douglas have investigated the selfassembly of chains in low-density Stockmayer fluids [18], regarding the process as a form of reversible polymerisation. These authors have also considered a natural extension of the Stockmayer potential to higher multipoles, in particular an LJ site plus point quadrupole [21, 22]. Quadrupole-quadrupole interactions favour the formation of extended two-dimensional sheets, which can produce tubes when the edges become connected. The LJ-plus-multipole class of potentials therefore provides control over the preference for compact (three-dimensional), sheet-like (two-dimensional) and chain-based (one-dimensional) structure in self-assembly. These tendencies compete with each other. In the present contribution we provide a systematic survey of the structure of small clusters of quadrupolar spheres in an attempt to identify and understand the structural motifs that emerge from the frustration between the isotropic and directional components of the potential.

\section{METHODS}

\section{A. Model potential}

The quadrupolar sphere is modelled as an isotropic Lennard-Jones site with a point quadrupole of variable strength superimposed [21, 23]. The pair potential, which we denote as LJQ, is of the form

$$
\begin{aligned}
V_{i j}\left(\mathbf{R}_{i j}, \Omega_{i}, \Omega_{j}\right)=4 u\left[\left(\frac{\sigma}{R_{i j}}\right)^{12}\right. & \left.-\left(\frac{\sigma}{R_{i j}}\right)^{6}\right] \\
& +V_{\mathrm{Q}}\left(\mathbf{R}_{i j}, \Omega_{i}, \Omega_{j}\right),
\end{aligned}
$$

where $\mathbf{R}_{i j}$ is the vector from particle $i$ to $j, R_{i j}$ is the magnitude of this vector, and $\Omega_{i}$ represents the orientational degrees of freedom of particle $i$. The parameters $u$ and $\sigma$ are the Lennard-Jones dimer equilibrium well depth and separation, respectively, and will be used as the units of energy and length henceforth. $V_{\mathrm{Q}}$ is the quadrupole-quadrupole interaction, which depends on the component(s) of the quadrupole tensor involved.

In this work, we consider the two quadrupolar arrangements of charges depicted in Fig. 1. In each case, the point quadrupole is reached by taking the limit in which the separation of the charges $d$ goes to zero, while the strength $Q=q d^{2}$ of the quadrupole moment is held fixed. For the linear arrangement of charges, the interaction between two point quadrupoles $i$ and $j$ can be written in terms of the unit vectors $\mathbf{e}_{i z}$ and $\mathbf{e}_{j z}$ along the body-fixed $z$-axes of the particles as

$$
\begin{aligned}
V_{\mathrm{Q}}^{\mathrm{lin}}= & 3\left(Q^{*}\right)^{2} u\left(\frac{\sigma}{R_{i j}}\right)^{5} \times \\
& \left(1+2 c_{z z}^{2}-20 c_{z z} r_{i z} r_{j z}-5 r_{i z}^{2}-5 r_{j z}^{2}+35 r_{i z}^{2} r_{j z}^{2}\right),
\end{aligned}
$$
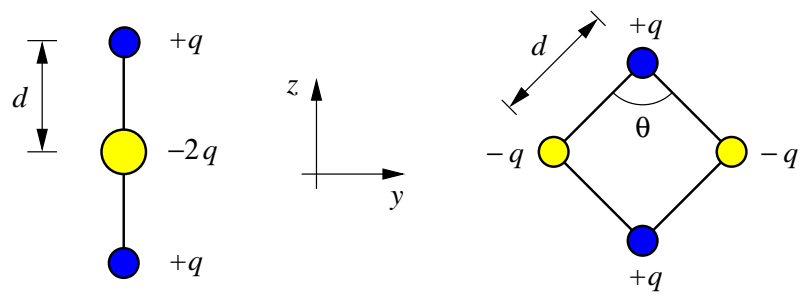

FIG. 1: Definition of the charge distributions in the "linear" (left) and "square" (right) quadrupoles with the local axis frame. The point quadrupole is obtained in each case by taking $d \rightarrow 0$ while keeping $Q=q d^{2}$ fixed.

where $c_{\alpha \beta}=\mathbf{e}_{i \alpha} \cdot \mathbf{e}_{j \beta}$, and $r_{i \alpha}=\mathbf{e}_{i \alpha} \cdot \mathbf{R}_{i j} / R_{i j}, r_{j \alpha}=$ $\mathbf{e}_{j \alpha} \cdot \mathbf{R}_{i j} / R_{i j}$ (note the sign convention with respect to the direction of $\mathbf{R}_{i j}$ ), with $\alpha$ and $\beta$ representing $x, y$ or $z$. The dimensionless parameter $Q^{*}=Q /\left(4 \pi \epsilon \sigma^{5} u\right)^{1 / 2}$ is the reduced quadrupole strength, $\epsilon$ being the dielectric permittivity of the medium. For the "linear" quadrupole defined by Eq.(2), we represent the orientation $\mathbf{e}_{i z}$ of the quadrupole using spherical polar angles.

In the $d \rightarrow 0$ limit, the pair interaction for the square arrangement of charges in Fig. 1 is

$$
\begin{aligned}
V_{\mathrm{Q}}^{\mathrm{squ}}= & \frac{3}{4}\left(Q^{*}\right)^{2} u\left(\frac{\sigma}{R_{i j}}\right)^{5}\left(2 c_{y y}^{2}-2 c_{y z}^{2}-2 c_{z y}^{2}+2 c_{z z}^{2}\right. \\
- & 20 c_{y y} r_{i y} r_{i y}+20 c_{y z} r_{i y} r_{j z}+20 c_{z y} r_{i z} r_{j y} \\
& -20 c_{z z} r_{i z} r_{j z}+35 r_{i y}^{2} r_{j y}^{2}-35 r_{i y}^{2} r_{j z}^{2} \\
& \left.-35 r_{i z}^{2} r_{j y}^{2}+35 r_{i z}^{2} r_{j z}^{2}\right) .
\end{aligned}
$$

Three variables are now required to specify the orientation of the quadrupole, and we have chosen to represent the vectors $\mathbf{e}_{i y}$ and $\mathbf{e}_{i z}$ in terms of Euler angles. The LJQ potential with $V_{Q}=V_{\mathrm{Q}}^{\mathrm{squ}}$ has received less attention [21, 22] in the past than LJQ with $V_{Q}=V_{\mathrm{Q}}^{\text {lin }}$.

The linear quadrupole corresponds directly to the spherical tensor component $Q_{20}$. The square quadrupole can be reached continuously from the linear arrangement by increasing the angle $\theta$ in Fig. 1 from 0 to $\pi / 2$, which corresponds to introducing a contribution from the component $Q_{22 c}$. At $\theta=\pi / 2$, the combination is $\frac{3}{2} Q_{20}+\frac{1}{2} \sqrt{3} Q_{22 c}$. The interactions between components of the quadrupole in the spherical tensor representation are tabulated in Appendix F of Ref. [24].

We will need to perform local geometry optimisations using the LJQ potentials, and have therefore derived and coded their analytic derivatives with respect to the Cartesian position coordinates and the angular orientational variables.

\section{B. Global optimisation}

We performed unbiased searches for the global minima of clusters bound by the LJQ potentials using the basinhopping algorithm [25], in which a Monte Carlo simu- 
lation is run on a transformed potential energy surface (PES) by performing a local minimisation of the energy at each step. The local minimisation [26] is key to the success of basin-hopping [27], and is a feature shared by other efficient methods of global optimisation for clusters, such as certain genetic algorithms [28].

For a given number of particles, $N$, and quadrupole strength, $Q^{*}$, several runs seeded from different random initial positions and orientations were performed. The number of Monte Carlo steps required to find a putative global minimum reliably in independent runs depends strongly on the size of the cluster and the strength of the quadrupole moment. It is also important to select a reasonable temperature for the accept/reject step in the basin-hopping runs. Although the success of the method is not very sensitive to the temperature, it must be high enough for the search to escape from local traps, but not so high that we fail to sample the low-lying minima in each region sufficiently. A fixed reduced temperature of $k T / u=1$ was often found to work well.

To generate a structural map for the clusters, it is necessary to explore the two-dimensional parameter space defined by the size of the cluster and the strength of the quadrupole. The straightforward approach of running basin-hopping on a grid of $Q^{*}$ points for each $N$ would be inefficient, since a small change in $Q^{*}$ will often lead only to a relaxation of the global minimum, with qualitative changes to a new structure occurring at larger intervals in $Q^{*}$. We have therefore devised a surveying scheme with an iterative element, designed to identify the values of $Q^{*}$ where the identity of the global minimum changes for a given $N$.

The algorithm begins with thorough searches for the global potential energy minimum at two values of the quadrupole strength, $Q_{\text {low }}^{*}$ and $Q_{\text {high }}^{*}$, that are far enough apart to lead to qualitatively different structures. These structures are then relaxed by local minimisation on a grid of $Q^{*}$ points that lie between $Q_{\text {low }}^{*}$ and $Q_{\text {high }}^{*}$, resulting in the correlation of the energy of each structure with $Q^{*}$. During this process, it is possible that one or both of the minima will disappear at some value of $Q^{*}$ due to a catastrophe in the PES [29], leading to a sudden change in energy as the structure falls into a different basin of attraction. In this case, a new basin-hopping run is performed at the $Q^{*}$ where the catastrophe occurred and the scan in $Q^{*}$ is continued.

Eventually, the energies of the relaxed structures initiated from $Q_{\text {low }}^{*}$ and $Q_{\text {high }}^{*}$ cross at some quadrupole strength $Q_{\text {cross }}^{*}$. New basin-hopping runs are then performed to identify the global minima at $Q_{\text {cross }}^{*} \pm \delta Q^{*}$ a little above and below the crossing point. If the basinhopping run at $Q_{\text {cross }}^{*}-\delta Q^{*}$ returns the same structure and energy as the relaxed structure from $Q_{\text {low }}^{*}$, then we assume that this structure was the global minimum not only at $Q_{\text {low }}^{*}$ and at $Q_{\text {cross }}^{*}-\delta Q^{*}$, but also at all values of $Q^{*}$ in the intervening range. In other words, we assume that there are no reentrant global minimum structures. This assumption is not only intuitively reasonable, given that $Q^{*}$ continuously changes the potential from isotropic van der Waals attraction to highly directional electrostatic interactions, but it is also borne out by careful checks of particular cases. The range $Q_{\text {cross }}^{*}+\delta Q^{*}$ to $Q_{\mathrm{high}}^{*}$ was treated analogously. Since a local relaxation is much faster than a full basin-hopping run, this procedure is far more efficient than using basin-hopping afresh at each intermediate $Q^{*}$ value.

If, on the other hand, the basin-hopping runs at $Q_{\text {cross }}^{*} \pm \delta Q^{*}$ return new structures with lower energy than the relaxed structures from $Q_{\text {low }}^{*}$ and $Q_{\text {high }}^{*}$, then this global minimum supersedes them and was in turn relaxed at values of $Q^{*}$ in both directions away from $Q_{\text {cross }}^{*}$ until the energy rose above those of the previous structures. A new check for the true global minimum must now be performed at this crossing point. The procedure was terminated when no lower minima were found at the crossing points of relaxed structures. Hence, full basinhopping runs need only be performed close to the locations where the identity of the global minimum changes.

\section{RESULTS}

\section{A. Local coordination of quadrupoles}

For a given separation $\mathbf{R}$, the energetically optimal arrangement of two point quadrupoles is with the local $y$ axis on one particle and the local $z$ axis on the other aligned with $\mathbf{R}$ and the other two local axes coplanar. This is true for any value of $\theta$ in Fig. 1, but we will refer to the arrangement as a "T-shape," which is most clearly seen for the linear case, $\theta=0$.

For the trimers, denoted $\mathrm{LQ}_{3}$ and $\mathrm{SQ}_{3}$ for the linear and square quadrupoles, respectively, triangular arrangements are optimal, as shown in the upper panels of Fig. 2. Despite the significant distortion away from three ideal T-shaped pair interactions, the energies of the trimers are each about 2.8 times the respective dimer energies at $Q^{*}=5$.

In the tetramers (lower panels of Fig. 2), the strain is relieved, making four undistorted T-shapes possible. For an interior angle of $45^{\circ}$, the slipped-parallel arrangement of the next-nearest neighbours is also favourable, further lowering the total energy of the tetramers to about 4.5 times that of the respective dimers at $Q^{*}=5$. An important difference between the linear and square quadrupoles is demonstrated by the tetramers. The axial arrangement of charges in the linear quadrupole means that rotation of a quadrupole about a local $z$ axis makes no difference to the energy. $\mathrm{LQ}_{4}$ is therefore able to lower its energy by twisting the quadrupolar axes slightly. This distortion places diagonally opposite particles above and below the plane of the projection in the lower-left panel of Fig. 2, allowing next-nearest neighbours to approach more closely. In contrast, $\mathrm{SQ}_{4}$ does not have this flexibility, and the structure in the lower-right panel of Fig. 2 is completely planar. 


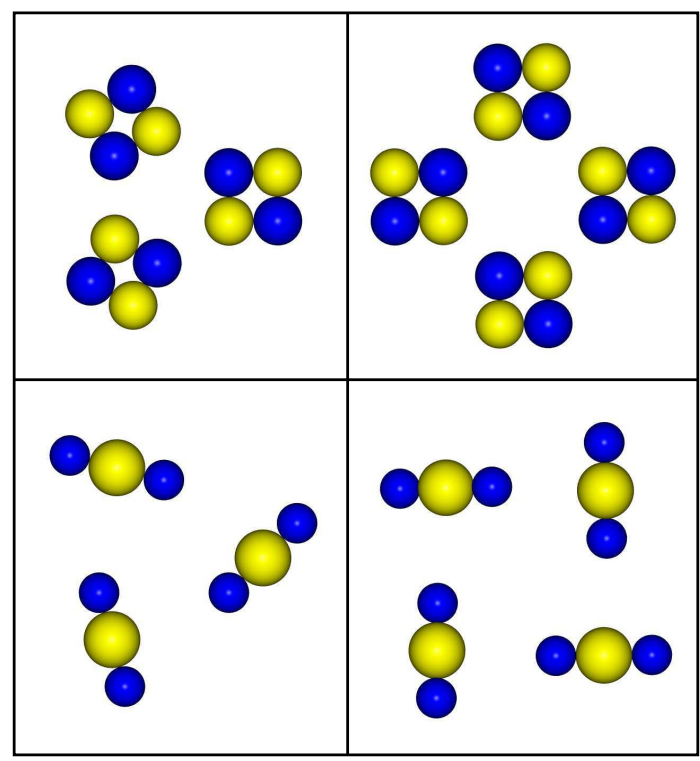

FIG. 2: Characteristic coordination motifs for the linear (left) and square (right) quadrupoles: global minima for the trimer (upper panels) and tetramer (lower) for a strong quadrupole (large $Q^{*}$ ). Extended charge arrangements are shown for illustration only; all calculations are in the point quadrupole limit. In the case of $\mathrm{LQ}_{4}$ (lower left), the quadrupole axes are not coplanar.

We note that the T-shape and slipped-parallel pair geometries are stationary points for dimers of the linear quadrupolar molecule carbon dioxide. However, in contrast to the LJQ model, the T-shape of $\left(\mathrm{CO}_{2}\right)_{2}$ is a saddle point, while the slipped-parallel geometry is stable [30].

\section{B. Strong and weak quadrupole limits}

The T-shaped nearest-neighbour geometry favoured by the quadrupole-quadrupole interactions encourages the formation of two-dimensional square networks. However, this tendency is frustrated by the LJ part of the potential, which drives the structure towards compact, highlycoordinated arrangements with polytetrahedral or icosahedral packing [25]. This competition produces a series of structural motifs that partially satisfy the two opposing trends. Fig. 3 summarises the structural maps of $\mathrm{SQ}_{N}$ and $\mathrm{LQ}_{N}$ as a function of the number $N$ of particles and the strength $Q^{*}$ of the quadrupole moment. Some structures are difficult to classify in an unambiguous or meaningful way, and such combinations of $N$ and $Q^{*}$ have been left blank in the figure for clarity.

For a sufficiently weak quadrupole moment, the global minimum must be close to the LJ global minimum structure, but with slight distortions induced by (a)
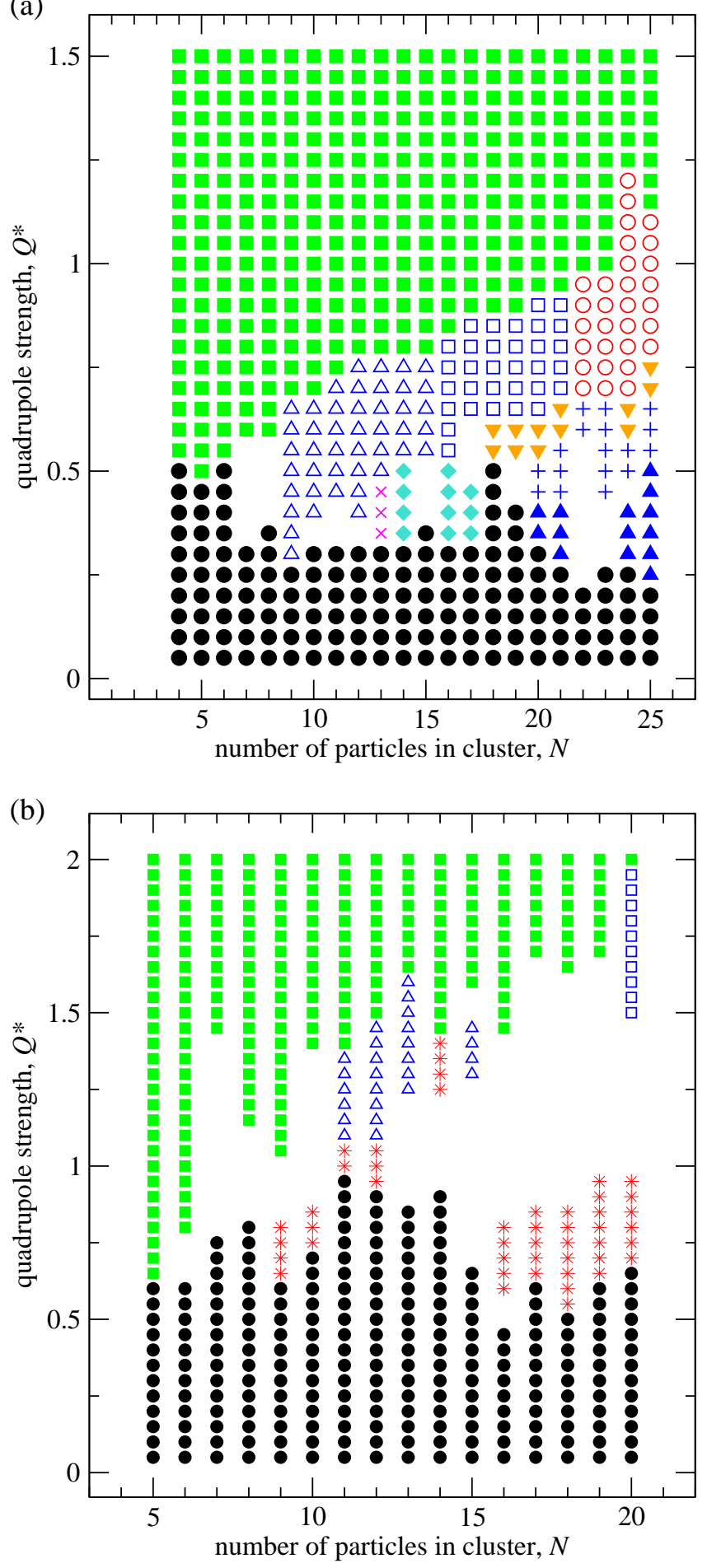

FIG. 3: Structural maps for (a) the square and (b) the linear quadrupole: relaxed LJ structure (filled circle), sheet (filled square), stacked triangular antiprisms (open upward triangle), decorated triangular antiprisms (filled upward triangle), stacked square antiprisms (open square), filled stacked pentagonal antiprisms (plus), hollow shell (open circle), filled shell (filled downward triangle), lattice-like (cross), decahedral core (filled diamonds), star (star). 
(a)
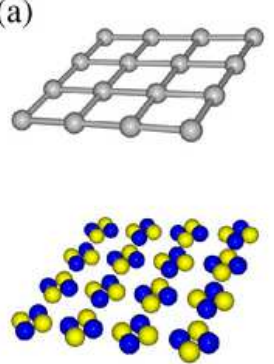

(d)
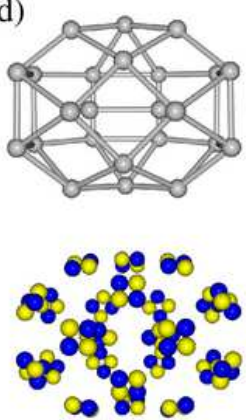

(b)

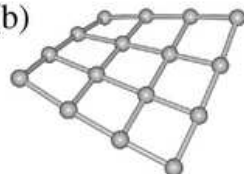

(c)

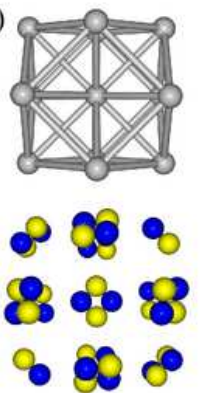

(e)
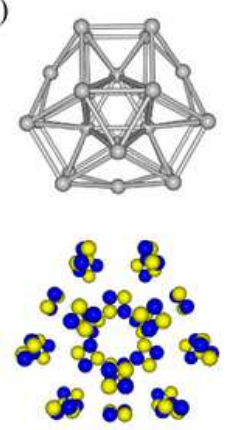

(f) (a)
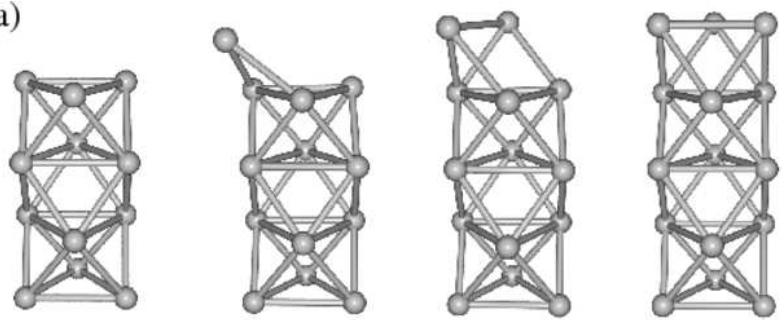

(b)
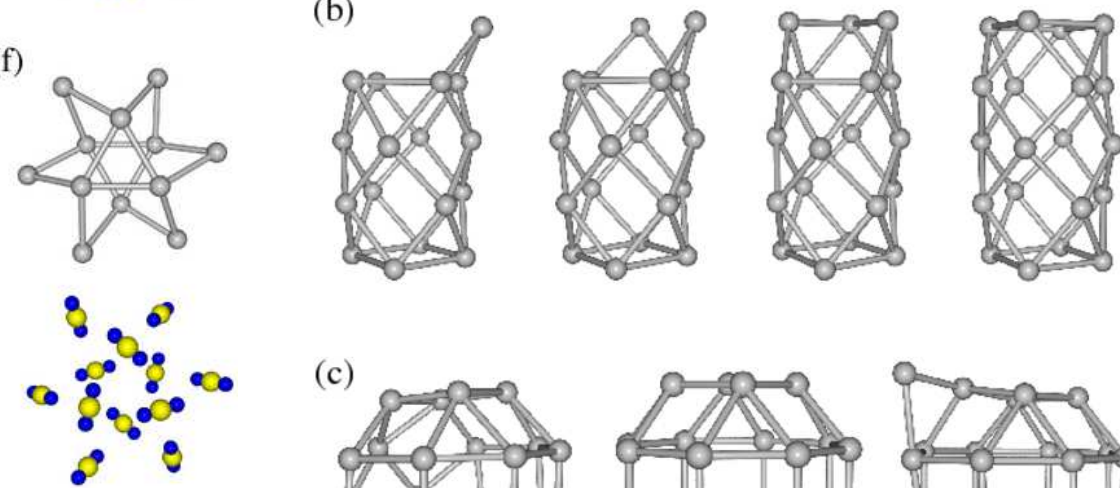

(c)

FIG. 4: Structures discussed in the text. (a) $\mathrm{SQ}_{16}$ sheet at $Q^{*}=2$, (b) $\mathrm{LQ}_{16}$ sheet at $Q^{*}=1.5$, (c) $\mathrm{SQ}_{13}$ cuboctahedron at $Q^{*}=0.4$, (d) $\mathrm{SQ}_{24}$ filled shell at $Q^{*}=0.6$, (e) $\mathrm{SQ}_{24}$ decorated triangular antiprisms at $Q^{*}=0.4$, (f) $\mathrm{LQ}_{12}$ star at $Q^{*}=1.025$.

the quadrupoles. However, effectively confining the quadrupoles to an icosahedral framework for $\mathrm{SQ}_{13}$ causes the quadrupoles to experience severe frustration, akin to that found in geometrically frustrated magnets 31. Hence, a given arrangement of the particles can correspond to multiple potential energy minima in the orientational part of configuration space, introducing a new source of complexity to the PES. The number of such isomers of the LJ structure generally increases with $Q^{*}$, but also depends sensitively and non-monotonically on $N$. For the near-icosahedral $\mathrm{SQ}_{13}$ there are two distinct isomers at $Q^{*}=0.025$, while for $\mathrm{SQ}_{19}$, where the global minimum is based on two interpenetrating icosahedra for this value of $Q^{*}$, we located 23 distinct arrangements by quenches of the LJ structure starting from random quadrupole orientations. This figure rises to several hundred for $\mathrm{SQ}_{19}$ at $Q^{*}=0.1$. Although these searches are not definitively exhaustive, the rapid increase in the number of isomers illustrates the roughness of the PES with respect to the orientational coordinates.

In the opposite limit of large $Q^{*}$, a two-dimensional sheet always emerges. The sheets for the square num-

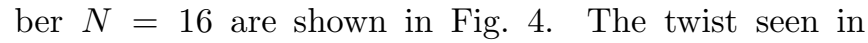
$\mathrm{LQ}_{4}$ is continued as the sheet grows, while the sheets of square quadrupoles are always planar. The sheet grows by adding particles at adjacent sites of the extended square lattice, with the dimensions of the lattice adapting to maximise the number of $\mathrm{T}$-shaped pairs in the
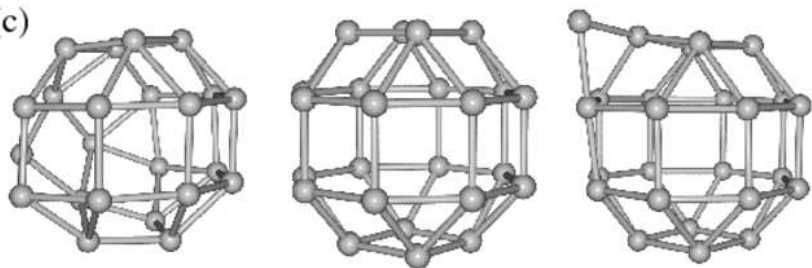

FIG. 5: Part of some growth sequences for the square quadrupole. (a) stacked triangular antiprisms, (b) stacked square antiprisms, (c) hollow shells.

first instance. It is often possible to achieve the maximum number of such pairs in more than one way on a square lattice, and next-nearest neighbour interactions then come into play. Hence, there can be close competition between structures even in the strong quadrupole limit. In contrast, the Stockmayer potential always has an unambiguous optimal structure consisting of a planar ring of head-to-tail dipoles in the strong dipole limit [20]. The lower boundary of the quadrupole sheet on the structural map moves (non-monotonically) to higher $Q^{*}$ as $N$ increases, because a larger number of LJ pair interactions must be disrupted to create the sheet. The widening region between the relaxed LJ cluster and the sheet is occupied by structures that strike a compromise between high-coordination number and sheet-like arrangements.

\section{The 13-particle cluster}

The 13-particle cluster, which for the pure LJ potential has a global minimum consisting of a centred icosahedron with point group $I_{h}$, provides a good illustration of the sequence of changes driven by the quadrupole in small clusters. For $\mathrm{SQ}_{13}$ with small $Q^{*}$, the quadrupole of the central particle aligns itself perpendicular to one of the icosahedral $C_{2}$ axes. The resulting small distor- 

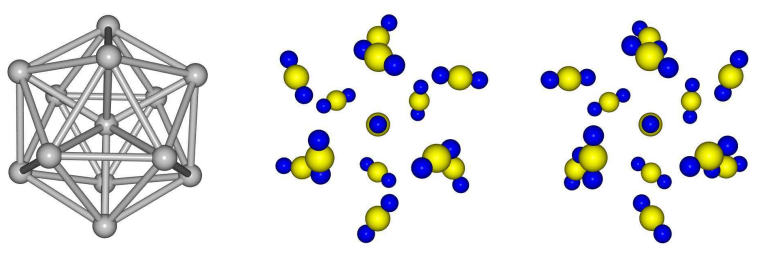

FIG. 6: The 13-particle icosahedron (point group $I_{h}$ ) viewed along a $C_{3}$ axis and the two $\mathrm{LQ}_{13}$ isomers at $Q^{*}=0.025$ of lowest energy. Both belong to point group $S_{6}$.

tions of the particle positions lower the symmetry to $C_{2 h}$. The quadrupole-quadrupole interactions are highly frustrated when confined to the vertices of the distorted icosahedron and at $Q^{*}=0.35$, the global minimum switches to a slightly distorted centred cuboctahedron $\left(D_{2 d}\right)$ - a fragment of face-centred cubic lattice (Fig. 4 r). This structure maintains the 12 -fold coordination of the central particle but the shell consists of squares and triangles, with quadrupole arrangements closer to those in the right-hand panels of Fig. 2, At $Q^{*}=0.525$, a second change occurs, to a stack of face-sharing triangular antiprisms (illustrated in the second panel of Fig. 5 a), which can also be regarded as a narrow tube if viewed down the three-fold axis. This structure sacrifices the high coordination of a more spherical lattice-like fragment for an elongated arrangement, in which the quadrupoles are better aligned. The switch to the sheet structure then takes place in two steps. First, at $Q^{*}=0.8$ a $3 \times 4$ sheet arises with the thirteenth particle in the same plane, bridging the central bond of a long side. At $Q^{*}=1.15$, the triangular face becomes too unfavourable, and the sheet adopts a $4 \times 4$ square with three of the corners missing.

A related sequence emerges with $Q^{*}$ for the cluster of 13 linear quadrupoles. Like the dipole moment in the 13particle Stockmayer cluster, the axis of the quadrupole on the central particle in $\mathrm{LQ}_{13}$ selects one of the $C_{3}$ axes of the icosahedron, here reducing the symmetry from $I_{h}$ to $S_{6}$. However, there are four other icosahedral minima differing by the quadrupole orientations. The two isomers with the lowest energy are illustrated in Fig. 6. With increasing $Q^{*}$, the cluster eventually passes to the stacked triangular antiprisms, but does so through a rather amorphous structure, unlike the highly symmetric cuboctahedron seen in $\mathrm{SQ}_{13}$. The linear quadrupole can tolerate a considerably higher $Q^{*}$ before switching to the sheet than can the square quadrupole - an observation that holds for all $N$ studied here.

\section{Structural families and growth sequences}

A number of structural families emerge in LJQ clusters and persist over some range of $Q^{*}$ and $N$. The stacked triangular antiprisms described above for 13 particles are seen for $\mathrm{SQ}_{N}$ with $9 \leq N \leq 15$. When $N$ is not a multi- ple of three, the end particles form an incomplete layer, giving rise to a simple growth sequence, part of which is depicted in Fig. 5h. This family does not continue indefinitely as the global minimum, but is replaced for $16 \leq N \leq 21$ by stacked square antiprisms (Fig. [5). The diamond-like faces on the surface of the square-based structure are flatter and closer to the ideal tetramer arrangement than those on the surface of the triangular antiprismatic stack.

Increasing the circumference of these stacks by another particle to make pentagons makes the diameter of the structure large enough to accommodate a line of particles down the centre of the stack, giving a filled tube-like arrangement. The additional contacts provided by the central line make such structures competitive at lower $Q^{*}$ than the stacked squares. In fact, pentagonal stacks arise in two forms in the structural map. The diamond symbols in Fig. 3(a) indicate clusters built around a decahedron, which contains a pentagonal prism. For pair potentials with a preferred nearest-neighbour separation, decahedral structures are less strained than icosahedral ones and are seen in the global minima of short-ranged isotropic potentials 32 and experimentally in metal clusters [33]. In LJQ clusters, the square faces are favoured by the quadrupolar interactions, and the structure can grow by building additional partial layers around the five-fold axis. Twisting the pentagonal layers gives the second type of pentagonal stack structure, filled pentagonal antiprisms, which appear on the map for $\mathrm{SQ}_{N}$ with $N \geq 20$. These tube-like stacks can be regarded as twodimensional sheets in which two opposite edges have been joined, thereby exchanging the energetic cost of an exposed edge for the penalty of curving the sheet. This trade-off is analogous to the formation of closed rings of dipoles 20]. Larger tubes have been observed to assemble spontaneously in the finite-temperature simulations of the LJQ fluid by Van Workum and Douglas [21].

Tubes can dispose of their remaining exposed edges by also closing the ends to make a shell. We observe hollow shells over a range of $Q^{*}$ in $\mathrm{SQ}_{22}$ and larger. For this class of structures, certain values of $N$ give rise to a structure of high symmetry. The first of these is $\mathrm{SQ}_{24}$, illustrated in the central panel of Fig. 5 , which has perfect $O_{h}$ octahedral symmetry. The shell normally grows by insertion of a particle into the surface, causing a distortion of the ideal triangular and square faces, but occasionally by the addition of an edge-bridging particle, as shown in Fig. 馬. The shell seems to be a permanent feature of larger $\mathrm{SQ}_{N}$ clusters. We have followed it as far as $N=36$, which forms an elongated shell of $D_{3 d}$ symmetry with triangular faces at the ends and antiprismatically stacked hexagons along the body.

If $Q^{*}$ is not sufficiently large, the shell is energetically penalised for its shortage of LJ nearest neighbour pairs. However, a large number of such pairs can be obtained by placing a few particles inside the shell. Hence, the hollow shell is typically preceded by a filled shell or filled pentagonal tube in the structural map, Fig. 3a. A shell 
encapsulating two particles is shown in Fig. $4 \mathrm{~d}$ for $\mathrm{SQ}_{24}$; compare the hollow shell for this cluster in the central panel of Fig. 5 .

Similar energetic compromises produce mixtures of structures that have already been described. For example, the transition from the LJ structure to the filled tubes and shells is sometimes bridged by decorated versions of the stacked triangular antiprisms, where the stack has been surrounded by a new layer. This arrangement is illustrated in a view down the three-fold axis for $\mathrm{SQ}_{24}$ in Fig. 4e. The characteristic network of square faces for the shell is beginning to emerge on the outside of these structures, but they maintain a larger number of LJ pairs than the filled shell.

The linear quadrupole tends not to give rise to hollow global minima. Although tube-like and shell-like structures do appear, they are collapsed into what would be the central space in the square quadrupole equivalents. This ability to distort, or inability to support a hollow interior, is a result of the axial symmetry of the linear quadrupole. A square array of linear quadrupoles, such as the one depicted in Fig. 4 b, can fold and twist along one of its diagonals without severely disrupting the $\mathrm{T}$ shaped nearest-neighbour interactions either side of the fold. This is not true of an array of square quadrupoles, such as that in Fig. 4a, in which each quadrupole defines a plane and not just a line. By collapsing inwards, the clusters of linear quadrupoles gain favourable interactions between opposite sides of the structure that would otherwise be held far apart. However, the collapsed structures are often rather amorphous, making them hard to classify or describe in a helpful way. For this reason, the structural map in Fig. 3] extends only to $\mathrm{LQ}_{20}$.

A distinctive and reproducible feature of the linear quadrupole clusters is a family of structures with a starlike organisation of the particles and a gear-wheel array of quadrupole axes, which are scattered around the structural map (Fig. 3b). Again, for particular values of $N$, the cluster can achieve a high symmetry that may be based on a three-fold or four-fold principal symmetry axis. An example belonging to point group $S_{6}$ is shown in Fig. 4f. However, the stability of these morphologies is strongly correlated with the number of particles; the stars are not observed away from the values of $N$ that allow the symmetry to be completed.

\section{CONCLUDING REMARKS}

The survey of putative global minima presented here shows that the competition between isotropic attractive forces and highly directional quadrupole-quadrupole interactions gives rise to a wide variety of structural motifs. These include elongated tube-like structures, hollow and filled shells, stars, and extended sheets. Some unusual point groups are represented in this collection.

Global optimisation is most challenging when the competing influences are closely balanced, i.e., for interme- diate strengths of the quadrupole in this work. Obtaining reproducible lowest-energy structures was significantly more difficult for the quadrupolar potential than for equivalent dipolar Stockmayer potential [20]. This observation, together with the multiplicity of minima that were found to have virtually identical positions but different orientations of the quadrupoles, hints at a complex potential energy surface in certain parts of the $\left(N, Q^{*}\right)$ parameter space. Confirmation and further exploration of this complexity would require a more comprehensive analysis of the energy landscape [34]. The landscape approach would provide information not only on the number of competing structures, but also on the barriers separating them and the rearrangement mechanisms that interconvert them. This information would provide a starting point for investigating the thermal stability and dynamic properties of the clusters, as well as the routes by which they might self-assemble.

The various families of structures have their own "magic" numbers at which a particular shape is complete and the landscape is probably minimally frustrated 14, 35]. Such numbers are well known for a variety of simpler interatomic potentials, and often correspond to the completion of successive icosahedral shells 36. at $N=13,55,147 \ldots$ The special stability associated with these sizes, combined with kinetic accessibility 14], can lead to prominent features such as experimental abundance [37]. In the present work, the tube-like structures of stacked triangular and square antiprisms achieve completed layers for multiples of three and four particles, respectively, while a shell can be elongated by the insertion of a complete hexagonal ring. In the strong quadrupole regime, sheets adopt defect-free squares when $N$ is a square number. It would be interesting to investigate whether these "perfect" structures are especially stable and self-assemble efficiently, as for magic number Lennard-Jones clusters [38].

We have seen that quadrupole-quadrupole interactions favour the formation of extended two-dimensional structures with four-fold coordination of the particles. In contrast, dipole-dipole interactions lead to extended pseudo one-dimensional chains, while isotropic attraction drives the structure towards compact three-dimensional arrangements. By careful balancing of the multipolar interactions, it should therefore be possible to exert considerable control over the structures that self-assemble out of multipolar particles with isotropic core interactions.

Briefly considering bulk phases, rather than finite clusters, such control could be useful in adjusting the networking properties of colloidal gels. For example, it has recently been shown that dipolar colloids can be encouraged to form more interconnected gel-like networks by a slight extension of the dipole [39]. From studies of models of patchy spheres with fixed maximum valency it is now known that the average coordination number of the particles in a gel has important consequences for the structure of the gel and for the underlying phase behaviour of the fluid from which it forms [40]. The present work 
suggests that the average coordination number could be finely tuned either by adding a weak point quadrupole to point dipolar particles, or by using a mixture of dipolar and quadrupolar spheres. Hence, multipolar particles could be an appealing alternative to patchy colloids for realizing and exploring reversible gels [41].

\section{Acknowledgments}

The authors are grateful to Josef O'Brien for some preliminary calculations on Lennard-Jones clusters with ex- tended quadrupolar distributions of point charges. MAM thanks EPSRC for financial support.
[1] S. C. Glotzer, M. J. Solomon, and N. A. Kotov, Amer. Inst. Chem. Eng. J. 50, 2978 (2004).

[2] M. R. Hoare and P. Pal, Nature Phys. Sci. 230, 5 (1971).

[3] O. Echt, K. Sattler, and E. Recknagel, Phys. Rev. Lett. 47, 1121 (1981).

[4] M. Dzugutov, Phys. Rev. A 46, R2984 (1992).

[5] J. P. K. Doye and D. J. Wales, Phys. Rev. Lett. 86, 5719 (2001).

[6] J. P. K. Doye, D. J. Wales, and S. I. Simdyankin, Faraday Discuss. 118, 159 (2001).

[7] S. Mossa, F. Sciortino, P. Tartaglia, and E. Zaccarelli, Langmuir 20, 10756 (2004).

[8] M. C. Rechtsman, F. H. Stillinger, and S. Torquato, Phys. Rev. E 75, 031403 (2007).

[9] F. H. C. Crick and J. D. Watson, Nature (London) 177, 473 (1956).

[10] J. B. Bancroft, G. J. Hills, and R. Markham, Virology 31, 354 (1967).

[11] D. Grueninger, N. Treiber, M. O. P. Ziegler, J. W. A. Koetter, M.-S. Schulze, and G. E. Schultz, Science 319, 206 (2008).

[12] D. C. Rapaport, Phys. Rev. E 70, 051905 (2004).

[13] D. J. Wales, Philos. Trans. Roy. Soc. Lond. A 363, 357 (2005).

[14] D. J. Wales and T. V. Bogdan, J. Phys. Chem. B 110, 20765 (2006).

[15] Z. Zhang and S. C. Glotzer, Nano Lett. 4, 1407 (2004).

[16] A. W. Wilber, J. P. K. Doye, A. A. Louis, E. G. Noya, M. A. Miller, and P. Wong, J. Chem. Phys. 127, 085106 (2007).

[17] A. S. Clarke and G. N. Patey, J. Chem. Phys. 100, 2213 (1994).

[18] K. Van Workum and J. F. Douglas, Phys. Rev. E 71, 031502 (2005).

[19] C. A. Oppenheimer and E. Curotto, J. Chem. Phys. 121, 6226 (2004).

[20] M. A. Miller and D. J. Wales, J. Phys. Chem. B 109, 23109 (2005).
[21] K. Van Workum and J. F. Douglas, Phys. Rev. E 73, 031502 (2006).

[22] J. F. Douglas and K. Van Workum, J. Mater. Res. 22, 19 (2007).

[23] S. F. O'Shea, G. S. Dubey, and J. C. Rasaiah, J. Chem. Phys. 107, 237 (1997).

[24] A. J. Stone, The Theory of Intermolecular Forces (Clarendon Press, Oxford, 1997).

[25] D. J. Wales and J. P. K. Doye, J. Phys. Chem. A 101, 5111 (1997).

[26] Z. Li and H. A. Scheraga, Proc. Natl. Acad. Sci. USA 84, 6611 (1987).

[27] D. J. Wales and H. A. Scheraga, Science 285, 1368 (1999).

[28] R. L. Johnston, Dalton Trans. pp. 4193-4207 (2003).

[29] D. J. Wales, Science 293, 2067 (2001).

[30] R. Bukowski, J. Sadlej, B. Jeziorski, P. Jankowski, K. Szalewicz, S. A. Kucharski, H. L. Williams, and B. M. Rice, J. Chem. Phys. 110, 3785 (1999).

[31] A. Harrison, J. Phys. Cond. Mat. 16, S553 (2004).

[32] J. P. K. Doye, D. J. Wales, and R. S. Berry, J. Chem. Phys. 103, 4234 (1995).

[33] L. D. Marks, Rep. Prog. Phys. 57, 603 (1994).

[34] D. J. Wales, J. P. K. Doye, M. A. Miller, P. N. Mortenson, and T. R. Walsh, Adv. Chem. Phys. 115, 1 (2000).

[35] J. D. Bryngelson and P. G. Wolynes, Proc. Natl. Acad. Sci. USA 84, 7524 (1987).

[36] A. C. Mackay, Acta. Crystallogr. 15, 916 (1962).

[37] J. Farges, M. F. de Feraudy, B. Raoult, and G. Torchet, Adv. Chem. Phys. 70, 45 (1988).

[38] J. P. K. Doye, M. A. Miller, and D. J. Wales, J. Chem. Phys. 111, 8417 (1999).

[39] R. Blaak, M. A. Miller, and J.-P. Hansen, Europhys. Lett. 78, 26002 (2007).

[40] E. Bianchi, J. Largo, P. Tartaglia, E. Zaccarelli, and F. Sciortino, Phys. Rev. Lett. 97, 168301 (2006).

[41] E. Zaccarelli, J. Phys. Cond. Mat. 19, 323101 (2007). 